\title{
ESTIMATION OF SOLAR RADIATION BY ARTIFICIAL NEURAL NETWORK MODEL
}

\author{
${ }^{1}$ SWETA KUMARI, ${ }^{2}$ KUMARI NAMRATA \\ ${ }^{1}$ M.Tech, Department of Electrical and Electronics Engineering, NIT Jamshedpur \\ ${ }^{2}$ Professor, Department of Electrical and Electronics Engineering, NIT Jamshedpur \\ E-mail: ${ }^{1}$ shwtakumari@gmail.com, ${ }^{2}$ namrata.ee@nitjsr.ac.in
}

\begin{abstract}
In this paper artificial neural Networks is used for predicting solar global radiation by using meteorological parameters. Seven meteorological parameters is considered for 14 cities to estimate solar radiation. The inputs are wind speed, rainfall, sunshine duration, temperature, vapour pressure, humidity, and rainy day. The ANN model is based on the feed forward multilayer perception model with seven inputs and one output. Based on the results, the average MAPE, mean bias error for the predicted global solar irradiation are $3.99 \%$ and $2.96 \%$. The results shown a better accuracy than other conventional prediction models. The results of these models have shown the capability of ANN models for predicting solar radiation in the locations that there is no any measurement.
\end{abstract}

Index Terms- Global Radiation, Solar Radiation, Artificial Neural Networks, Meteorological Parameters, Forecasting.

\section{INTRODUCTION}

Solar energy is the portion of the sun's energy available at the earth's surface for useful applications, such as raising the temperature of water or exciting electrons in a photovoltaic cell, in addition to supplying energy to natural processes like photosynthesis. This energy is free, clean and abundant in most places throughout the year. Its effective harnessing and use are of importance to the world, especially at a time of high fossil fuel costs and the degradation of the atmosphere by the use of these fossil fuels. Availability of solar radiation on horizontal surface is essential for the optimum design and study of solar energy systems. The traditional way of knowing the amount of global solar radiation (GSR) in a particular region is to install pyranometers at as many locations as possible in this region thus requiring daily maintenance and data recording, and consequently increasing cost of GSR data collection[2]. Therefore, it is rather more economical to develop methods to estimate the GSR using climatological parameters. Solar radiation data provide information on how much of the sun's energy strikes a surface at a region on earth, during a particular time period. In developing countries, GSR measurements are usually made at few sites, because there are not many solar observation stations, as well as expensive equipments are required to achieve solar radiation quantity[3].

Using artificial neural network as an estimation tool has proved its efficiency in predicting different parameters via other parameters that their relationship is not specified. Climatological and meteorological parameters are important parameters in indicating the amount of solar radiation in a selected region. So applying artificial neural networks can be valuable in determining the effects of meteorological parameters and finally prediction of solar radiation. Data from 14 cities was used in training the $\mathrm{NN}$ and the remaining data was used for testing. The inputs are wind speed, rainfall, sunshine duration, temperature, vapour pressure, humidity and rainy day. In this study more climatological indicators have been used for predicting solar radiation that it can provide better results in outputs of the model.

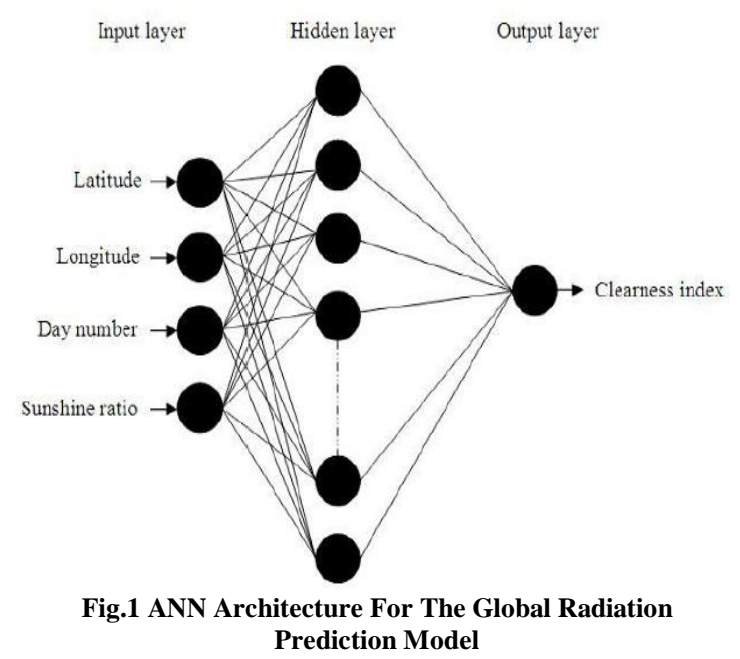

\section{MATHEMATICAL MODELLING OF ANN}

Solar radiation is classified in two main parts, the extraterrestrial solar irradiation $\left(E_{\text {extra }}\right)$ and the global solar irradiation. The variable ( $\left.E_{\text {extra }}\right)$ stands for the total solar energy above the atmosphere while $\left(E_{t}\right)$ is the total solar energy under the atmosphere. The value of $E_{\text {extra }}$ is given

$$
E_{\text {extra }}=\left\{I_{o}[1+0.34 \cos (2 \pi N / 365)]^{*}\right. \text { Day...... }
$$


Where $I_{o}$ is the solar constant, $1,367 \mathrm{w} / \mathrm{m}^{2}$ and $\mathrm{N}$ is the number of the day. The day length is calculated by

Daylength $=2 / 15 \cos ^{-1}(\tan L \tan \partial)$.

Where $\mathrm{L}$ is the latitude and $\partial$ is the angle of declination, given by

$\partial=23.45 \sin [360 *(284+N) / 365]$

The global solar irradiation ( $E_{T}$ ) on a tilted

surface consists of three parts

$E_{T}=E_{B}+E_{D}+E_{R}$

Where $E_{B} E_{D}, E_{R}$ are beam (direct), diffused and reflected solar irradiation, respectively. On a horizontal surface $E_{R}$ is equal to zero; therefore $E_{T}$ on a horizontal surface is given by

$$
E_{T}=E_{B}+E_{D}
$$

The global $E_{T}$ can be calculated using $E_{\text {extra }}$ as below

$$
E_{T} / E_{\text {extra }}=K_{T}
$$

Where $K_{T}$ is clearness index which will give the predicted solar radiation.

\section{DESIGNING OF ARTIFICIAL NEURAL NETWORK}

Designing ANN models follows a number of systemic procedures. In general, there are five basics steps:

(A) collecting data, (B) pre-processing data, (C) building the network, (D) train and (E) test performance of model.

\section{(A) DATA COLLECTION}

Collecting and preparing sample data is the first step in designing ANN models. Measurement data of wind speed, rainfall, sunshine hour, temperature, vapour-pressure for 14 cities (Ranchi, thiruvananthapuram,Bangalore,Portblair chennai,Goa,Bhavnagar,Kolkata,Ahmdabad,Jodhpu r,Jaipur New delhi,Srinagar) in India.

\section{(B) DATA PRE-PROCESSING}

After data collection, three data pre-processing procedures are conducted to train the ANNs more efficiently. These procedures are: (1) solve the problem of missing data, (2) normalize data and (3) randomize data. The missing data are replaced by the average of neighbouring values during the same week.

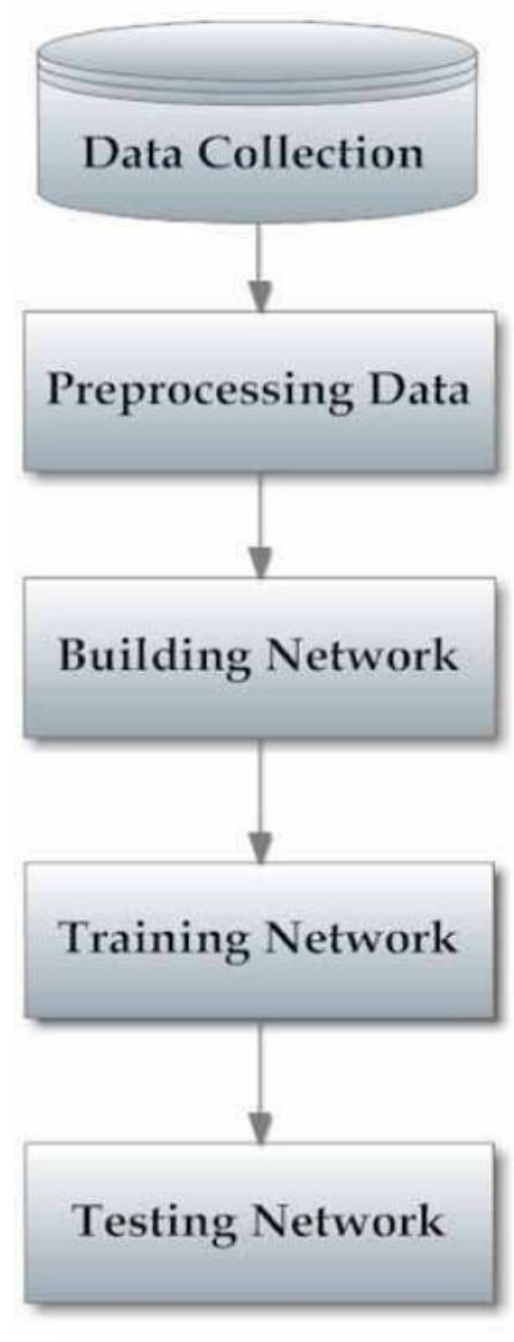

\section{(C) BUILDING THE NETWORK}

At this stage, the designer specifies the number of hidden layers, neurons in each layer, transfer function in each layer, training function, weight/bias learning function, and performance function. In this work, multilayer perceptron (MLP) and radial basis function (RBF) networks are used.

\section{(D) TRAINING THE NETWORK}

During the training process, the weights are adjusted in order to make the actual output (predicated) close to the target (measured) outputs of the network.

\section{(E)TESTING THE NETWORK}

At this stage unseen data are exposed to the model. In order to evaluate the performance of the developed ANN models quantitatively and verify whether there is any underlying trend in performance of ANN models, the root mean square error (RMSE) and the mean bias error (MBE) were conducted. RMSE provides information on the short term performance which is a measure of the variation of predicated values around the measured 
data. The lower the RMSE, the more accurate is the estimation. MBE is an

indication of the average deviation of the predicted values from the corresponding measured data and can provide information on long term performance of the models; the lower MBE the better is the long term model prediction. A positive MBE value indicates the amount of overestimation in the predicated GSR.

$$
\begin{aligned}
& \mathrm{MBE}=1 / n \sum_{i=1}^{n}\left(I_{p, i}-I_{i}\right) \ldots \ldots . . \\
& \mathrm{RMSE}=\sqrt{1 / n \sum_{i=1}^{n}\left(I_{p, i}-I_{i}\right) . .}
\end{aligned}
$$

\section{IV.RESULTS AND DISCUSSIONS}

To ensure efficiency of developed network.14 sites is chosen fig. 3 shows the predicted clearness index compared with the measured value. The figure shows the good agreement between measurement and prediction. The average mean absolute percentage error in predicting the global solar radiation is $3.99 \%$ and mean bias error is 2.63\%.Mean bias error is indication of the average deviation of the predicted values from the measured values. A Positive MBE values indicate the amount of overestimation in the predicted global solar radiation. RMSE provides information on the short term performance and is a measure of the variation of the predicted values around the measured data, indicated by the scattering of data around the linear lines.

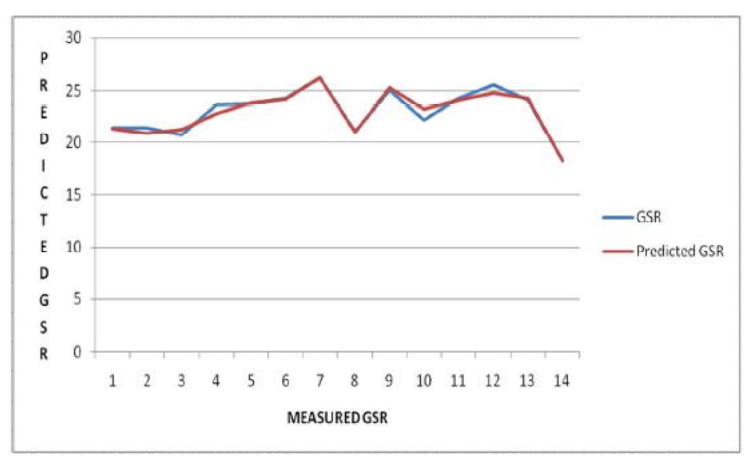

Fig.3 Measured GSR Versus Predicted GSR Of 14 Cities

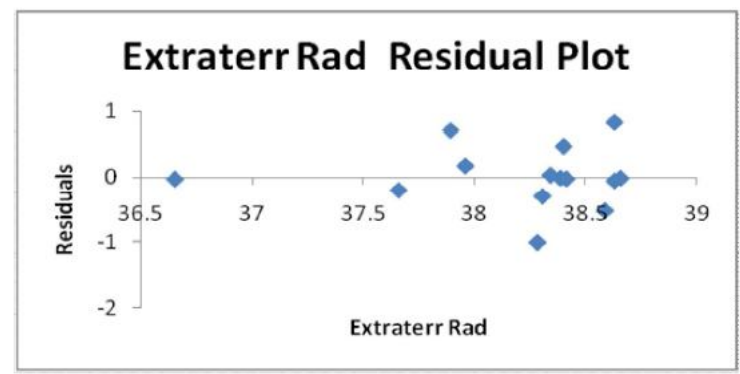

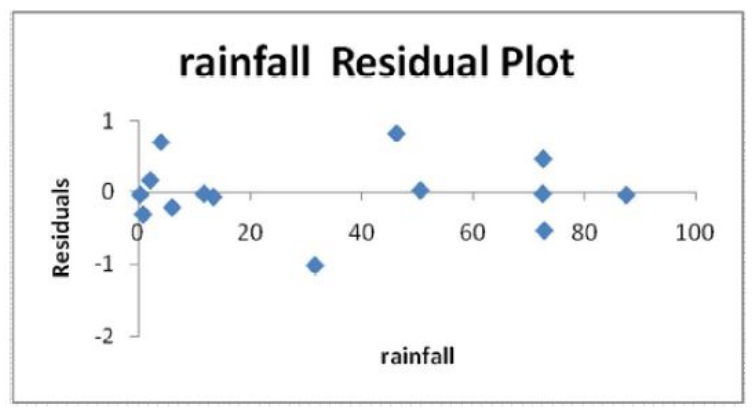
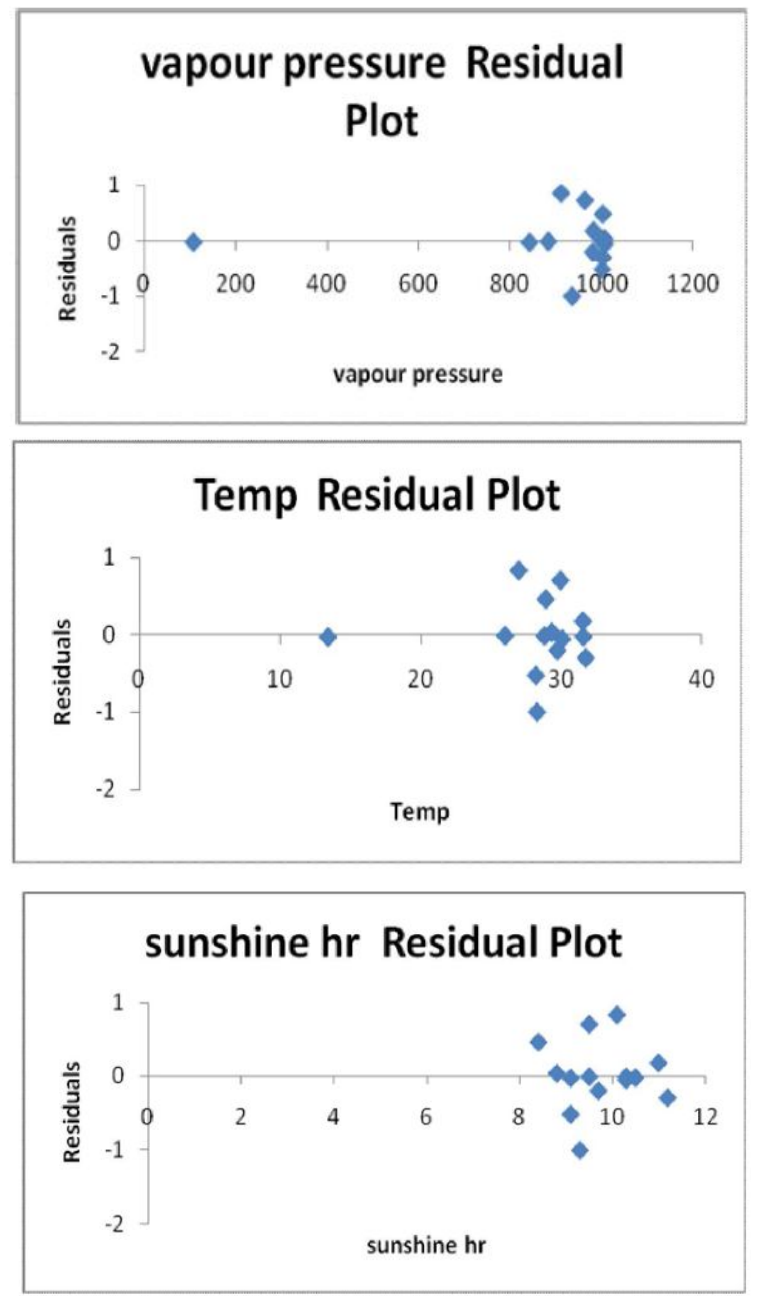

Fig.4 Relation between different climatological parameters and residuals

\section{CONCLUSIONS}

Several benefits of solar energy have made it as an important energy production tool. But using this energy requires having knowledge about radiation potential in different locations. Recently the use of artificial neural networks has developed widely prediction tool. In this paper we have used an ANN model for predicting solar global radiation in 14 different cities of India. For this model numbers of climatological were introduced and applied for predicting solar radiation. These parameters are the most conventional parameters that are available in many places. Application of this model shows better 
results rather than traditional prediction models and it could be used to evaluate the solar potential of a location. The use of these models in the remote locations that solar measurement devices are not available can be beneficial as an effective tool to select the most efficient locations for using solar energy.

\section{REFERENCES}

[1] Elizondo D, Hoogenboom G, Mcclendon RW, "Development of a neural network model to predict daily solar radiation," Agric Forest Meteorol, 1994; 71:115-32.

[2] Sozen A, Arcakly' ogblu E, Ozalp M, Kany' t EG, “ Use of artificial neural networks for mapping the solar potential in Turkey," Appl Energy, 2004;77:273-86.

[3] Reddy KS, Manish R. , "Solar resource estimation using artificial neural and comparison with other correlation models," Energy Convers Manage 2003; 44:2519-30.
[4] Tymvios FS, Jacovides CP, Michaelides SC, Scouteli C. "Comparative study of Angstroms and artificial neural networks methodologies in estimating global solar radiation," Sol Energy 2005; 78: 752-62.

[5] Lo' pez G, Batlles FJ, Tovar-Pescador J. " Selection of input parameters to model direct solar irradiance by using artificial neural networks," Energy2005; 30:1675-84.

[6] Hontoria L, Aguilera J, Riesco J, Zufiria P. "Recurrent neural supervised models for generating solar radiation synthetic series,” J Intell Robot Syst 2001; 31:201-21.

[7] Hontoria L, Aguilera J, Zufiria P. "Generation of hourly irradiation synthetic series using the neural network multilayer Perceptron" Solar Energy 2002;72(5):4416.

[8] Reddy KS, Manish R. "Solar resource estimation using artificial neural networks and comparison with other correlation models," Energy Convers Manage 2003; 44:2519_ 30 .

[9] Mihalakakou G, Santamouris M, Asimakopoulos DN. "The total solar radiation time series simulation in Athens, using neural networks," Theory Appl Climatol 2000; 66:185-97.

[10] Alam S, Kaushik SC, Garg SN. "Computation of beam solar radiation at normal incidence using artificial neural network," Renew Energy 2006; 31:1483-91. 